\title{
Training Effectiveness: The Case of Quality and Food Safety Training at ABC Company
}

\author{
G.R.V.C. Nissanka ${ }^{1}$ and Kumudinei Dissanayake ${ }^{2}$ \\ ${ }^{\mathbf{1}}$ Faculty of Management \& Finance, University of Colombo \\ ${ }^{2}$ Faculty of Management \& Finance, University of Colombo
}

\begin{abstract}
In light of the evidence on ineffectiveness of existing quality and food safety trainings at the $\mathrm{ABC}$ company, this study explored the reasons and possible actions for improving the effectiveness of the training programs. The study was designed to cover the entire training process of quality and food safety in a 360 degrees evaluation with the use of the Kirkpatrick's training evaluation model. The data were gathered from the trainees, trainers, department heads and the human resource department by means of a questionnaire survey and structured interviews. The study covered 65 respondents altogether. The results show the existence of significant gaps in all the steps of the training process of quality and food safety. The gaps are lying in the processes of training needs identification, establishing the training objectives, selecting the training methods, arranging the trainings and evaluating the performances of the trained people. These deficiencies were triggered by the non-availability of a comprehensive training management protocol in $\mathrm{ABC}$ Company. It is implied that the identification of training and development as a subsystem would reduce the ineffectiveness of the quality and food safety trainings of the company.
\end{abstract}

Keywords: Food safety; Kirkpatrick's model; Quality; Training effectiveness 


\section{Introduction}

Sustainability of a firm in the food industry is usually challenged due to the fast-moving nature of products and easy entry of new product options. As such, becoming the market leader and maintaining the leadership position are both hard for a firm in the food industry. However, any organization can gain the superiority over the market leader by redefining the market through improving the quality of the goods or services by becoming the quality leader in the eyes of the customer (De Feo \& Juran, 2010). This is an experienced reality in the food industry as in all other industries.

The human factor which involves in the food manufacturing process plays a major role in assuring and maintaining the attained quality of a product. Thus, people are the most important factor in assuring quality of products and services, and managing the human factor has a significant impact on the quality performance of an organization (Daud, Jamaludin, \& Ramanr, 2012). The top performing companies attempt to assure a strong quality culture with every employee those who understand the importance of quality and work to improve it (Spindelndreier \& Lesmeister, 2012). The need of encouraging employees to develop the awareness about achieving the set quality objectives in the food industry has been highlighted in the previous studies. As stated by Antic and Bogetic (2015), expanding the awareness of the quality of products among employees should be above all other responsibilities of the management.

The case of a Sri Lankan company (pseudonym: ABC Company) has opened up the avenue for probing into the human involvement in the quality of its products. The ABC Company is engaged in manufacturing 
of highly perishable food products. In the organization, as per the $2018 / 19$ budget, the total number of employees is 425. Being a manufacturer of highly perishable food products, achieving the higher quality and food safety standards and maintaining the same are highly significant matters in the entire manufacturing process of this company. With all the improved and updated systems implemented to assure the quality and food safety, maintaining, updating and improving the systems according to the industry standards have been managed by people in the organization.

During the past few years, some of the monitored quality and food safety indicators of $\mathrm{ABC}$ Company have not indicated the improvements on its own measures and some further deteriorations could be observed in the results. During the informal discussion conducted among the senior managers of ABC Company, the major concern raised about this issue was about the return on investment on the training of the plant employees on the quality and food safety. The training programs of quality and food safety covers the employees in all the departments to assure the compliances related to the quality and safety of the food products. However, it was noted that the measurable indicators do not reflect the improving attitudes towards the expected product quality and food safety.

Total 143 employees (both executive and non-executive grades) out of the total carder (425 employees) have attended the quality and food safety trainings (Source: 2018/19 budget figures of ABC Company). The training programs on quality and food safety have been arranged during the past several years. There have been conducted a number of training programs (such as ISO9001, HACCP, ISO22000, Personal 
Hygiene, GMP, food safety awareness) and seven quality assurance training programs conducted by the company during the financial years 2015/2016 and 2016/2017. Three out of the seven programs had been conducted by an external training institute and the rest of the four programs had been arranged internally by the internal trainers.

However, surprisingly, the GMP (Good Manufacturing Practices) audit score has been low and declining in the company. In addition to this, the number of process non-conformities reported is on the rise and the trend of the customer complaints is also increasing. Most significantly, the increase of manufacturing volume does not justify the increase of these numbers. The Table 1 below depicts some of the quality indicators.

Table 1: Quality and Food Safety indicators in year 2015/16, 2016/17, 2017/18

\begin{tabular}{|l|l|l|l|}
\hline Quality \& Food Safety Indicators & $\mathbf{2 0 1 5 / 1 6}$ & $\mathbf{2 0 1 6 / 1 7}$ & $\mathbf{2 0 1 7 / 1 8}$ \\
\hline No. of Consumer Complaints & 47 & 64 & 61 \\
\hline No. of Process Non-Conformities & 78 & 106 & 150 \\
\hline GMP Audit Score & $69 \%$ & $58 \%$ & $55 \%$ \\
\hline
\end{tabular}

Source: Adapted from the KPI Records of Department of Quality Assurance, ABC Company (Year 2015/16, 2016/17, 2017/18)

Thus, despite the efforts made by the ABC Company to conduct quality and food safety training programs in the recent past, the deterioration of the quality and food safety indicators has led to a puzzle which needs to be addressed immediately. At this backdrop, there is a reasonable concern about the effectiveness of the quality assurance training programs conducted by the ABC Company for its plant employees. Accordingly, the research questions addressed in this study are two-fold: 
(a) why have the quality and food safety training programs conducted by the $\mathrm{ABC}$ company not been effective, and (b) how can the $\mathrm{ABC}$ Company improve the effectiveness of the quality and food safety trainings arranged for its plant employees. In line with the above research questions, the objectives of the study are to explore the reasons why have the quality and food safety training programs conducted by the $\mathrm{ABC}$ company not been effective, and how can the ABC Company improve the effectiveness of the quality and food safety trainings arranged for its plant employees.

The literature review revealed that there is a dearth of studies pertaining to the factors affecting the effectiveness training programs on quality and food safety of the food manufacturing industry in Sri Lanka. Hence, the findings of this investigation could be utilized by the management of the ABC Company as well as similar other companies in the same industry. The findings of the study would be beneficial to the human resource development team, especially in optimizing the training effectiveness of the food industry. The findings of this research could also serve as a source of information for further studies to be carried out in the Sri Lankan food industry while serving as a guide for manufacturers in other industries when organizing the effective training programs for their employees.

\section{Literature review}

\section{Quality and food safety}

Quality and food safety are two major global concerns connected with various areas of everyday life as well as a special concern for the food manufacturing companies. Food safety has been defined as the handling, preparing and storing food in a way to minimize a possible risk of 
individuals becoming sick from food-borne illnesses (Santacruz, 2016, p.1). Thus, both quality and food safety are equally important in the food industry, as the quality assures the meeting of customer preference and the food safety assures the safe food to the customer.

It is essential for a food manufacturing company to maintain the quality of their products and follow the standard food safety principals in order to become the best marketer in the industry. In order for a company to do so it is an adequate necessity to conduct training programs to enhance the productivity and the effectiveness of both executives and the non-executives of the company. There are research findings that adequate food safety training for all employees can have a positive impact on health inspection scores and on some food safety behaviors (Rowell et al. 2013, p. 2). As per Rowell et al. (2013), the basic hygiene requirements such as hand washing are considered in relation to the food safety, while the employee training and implementation are essential in preventing food-borne illness. They further suggest that these trainings do not only improve employee knowledge, but also provide employees the needed behavior-based learning to change their actions. Although the quality and food safety management are the system driven processes, the people who manage these processes should have been well developed to change their attitudes towards quality and food safety. The quality is explained as an attitude. The attitudes are adopted and not innate, and at the same time, attitudes not born but learned (Dewsnap, n.d.). Hence, attitudes towards the quality should be enhanced in the human factor in an organization. Training plays a major role in this regard. 


\section{Training for quality of food safety}

As elaborated by Gaungoo and Jeewon (2013), "Training of managers, supervisors and all people who can influence the safety of food is essential to reduce the unacceptable high levels of food poisoning" ( $p$. 2). Training is not only for acquiring knowledge. It is about sharpening the skills, changing the behavior to do the things better or differently than that of the same was performed. "Business concepts-human resources" (n.d.) notes that training and development are a subsystem of an organization which maintains the improvement of the performance of individuals and groups of the organization. It identifies training as an educational process which sharpens the skills, knowledge, and changes the attitudes required in developing the employees.

As stated by Armstrong (2009), training involves "the application of formal processes to impart knowledge and help people to acquire the skills necessary for them to perform their jobs satisfactorily" (p. 665). According to Spindelndreier and Lesmeister (2012), training is intended to modify or develop knowledge, skills and attitude through learning experiences and to achieve effective performance in an activity or range of activities. Training can be offered as skill development for individuals and groups with an ultimate requirement to modify their attitudes.

By conducting effective training programs, organizations can evaluate the growth of its employees' efficiency and the quality of its products or services. It also provides the organization with the ability to survive, especially with the hard competition of the days. In general, training is a typical process to provide employees with new skills or improve their 
knowledge to a higher stage in order to enhance the behavioral change to alter the culture of the organization to achieve the set goals.

\section{Types of Training and learning}

There are two main types of trainings that should be considered in organizations (Spilsbury, 1995). They are on-the-job training and offthe-job training. As explained by Spilsbury (1995), in on-the-job trainings, employees learn in an environment in which they will need to practice the knowledge, skills competencies that are needed for employees to perform a specific job within the workplace and work environment. On-the-job training uses the regular or existing workplace tools, machines, documents, equipment, knowledge, and skills necessary for an employee to learn to effectively perform his or her job. The offthe-job training is the training method where the employees learn their job roles away from the actual work floor. The off-job trainings are performed in a separate venue which is out of the work place in the same organization or outside. In these trainings the practice approaches may be limited.

In addition to these, the initial categorization of learning at workplace is identified as informal learning and formal learning (Armstrong, 2009). Informal learning is acquired through various methods including conversation, discussions, guidance or social interaction with other colleagues, teamwork and mentoring. It takes place while people are learning on-the-job. Employees can learn and acquire 70 percent of knowledge, information about their job in an informal way via the processes which are not structured or sponsored by the organization. Formal learning is a planned and systematic learning process. It makes use of structured training programs consisting of instruction and practice 
that may be conducted on or off the job. Formal learning and developmental activities may be used such as action learning, coaching, mentoring and outdoor learning. Both the quality and food safety trainings can be formal or informal and on-the-job or off-the-job.

\section{Training cycle}

Spilsbury (1995) suggested that the training should not be considered as a static event or a liner process but as a cyclical process. Accordingly, the information about the effectiveness is fed-back into the design of the training program. For this reason, the training process was called the 'training cycle'. "The Training Cycle consists of a series of steps which lead to a training event being undertaken: evaluation provides feedback which links back to the initial stages of training design" (Spilsbury, 1995, p. 19). Thus, the training cycle is designed before the training program is conducted and continues after the program has been completed. The figure 1 below illustrates the five stages of the Training Cycle.

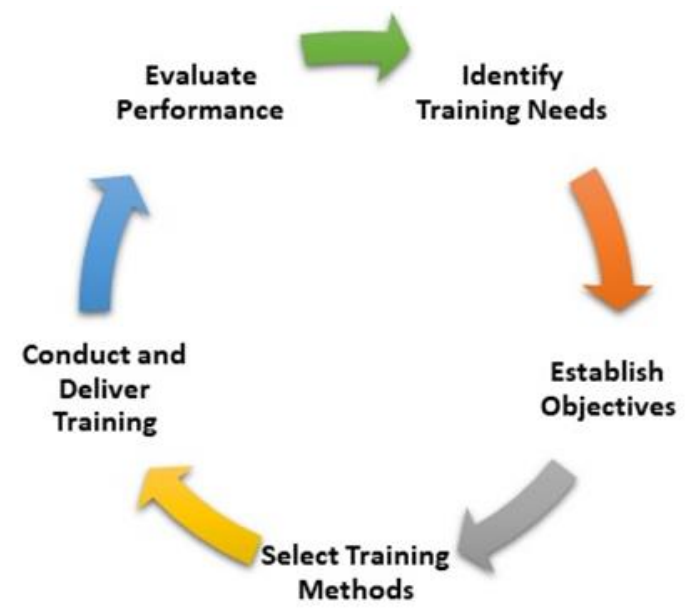

Figure 1: Training cycle

Source: Adopted from Biech, (n.d.) 
The steps of the training cycle can be explained as below (Donald Kirkpatrick's Learning Evaluation Model 1959; Review and Contextual Material).

1. Identify training needs - Conduct an assessment and analyze the data, to identify specific areas of needs for the training. The content for the training and the method of training should be developed.

2. Establish objectives - State exactly what the training should accomplish. It can be either or both learning/performance objective and training objective.

3. Select Training methods - Decide on the suitable methods accordingly to the needs and objectives.

4. Conduct and deliver Training - Conduct with adequate preparation with professional trainers and good presentation skills. Be more of a facilitator than an instructor.

5. Evaluate Performance - The evaluation stage is an important part of the training cycle. This would indicate whether the objectives were reached, the progress on the trained and the highlights areas for future training needs.

The steps of the training cycle have been explained differently in various studies. However, the five-step training cycle is commonly used.

\section{Training effectiveness}

Bramley (1997) defined training effectiveness as "a measure that examines the degree to which training improved the employee's knowledge, skill, and behavioral pattern within the organization as a result of the training" (p. 20). The effectiveness of training can be fundamentally measured by evaluating the employee's performance within a selected period of time after the training is conducted 
(Morrison \& Hammon, 2000). Chong (2006) stated that the evaluation of training effectiveness is usually connected to measuring the change occurred which ultimately leads to performance (p. 1). Thus, in a synthesized view, Bramley (1997), Chong (2006) and Morrison and Hammon (2000) depict that the measurement of training effectiveness includes gain of skills or knowledge, changing the behavioral pattern or performances of the people trained. Therefore, each step of the training cycle (Spilsbury, 1995) should be evaluated and assured to achieve the above result which is expected from the trained employees. Another major aspect of transfer of knowledge was pointed by Kjeldsen (2006) highlighting the requirement of examining the level of newly acquired knowledge, skills or behaviour retained in a longer period of time. Kraiger, Ford, and Salas (n.d.) also argued that the performances shown by trainees after a training at a particular level in the short run may differ substantially in the long run.

\section{Evaluating the training effectiveness}

Measuring or evaluating the effectiveness of training of an organization could bring several benefits. When the training session is planned, it should start with a list of specific learning objectives which should be the starting point for the measurement. Depending on the objectives and depending on whether it intended any changes in knowledge, skills, or attitudes, the learning can be measured ("Kirkpatrick's Four-Level Training Evaluation Model," n.d.). Evaluating the training effectiveness is a difficult and costly task. Before setting up structures to measure the training effectiveness, it is required to make sure whether there is a serious interest in training because the benefits of conducting evaluations can be substantially outweigh the cost incurred for it 
(Spilsbury, 1995). Evaluating the training outcomes in the absence of an evaluation of the full process of the training cycle, is also cost effective, however, this approach does not provide the full picture of the effectiveness of the entire training process.

\section{The training evaluation cycle}

The five-steps training evaluation cycle, presented by Kirkpatrick, has been well used in the existing literature ("Manual on Training Evaluation," n.d.). This process can be briefed as below:

\section{Identify the Purposes of Evaluation.}

Before developing evaluation systems, the purposes of evaluation must be determined.

\section{Select Evaluation Method}

The commonly used evaluation model is Kirkpatrick's four levels of training evaluating program.

\section{Design Evaluation Tools}

Various evaluation tools can be selected depending on the purposes and methods of evaluation such as questionnaire, interviews, focus group discussions etc.,

\section{Collect Data}

It should be decided who, when and how the data are collected.

\section{Analyze and Report Results}

This step includes the analysis of collected data for arriving at evaluation results and reporting them to the required parties. 


\section{Models of training evaluation}

The most popular and widely used training evaluation model is the Kirkpatrick Model. This model has been introduced by Donald Kirkpatrick in 1950. It has been undergone some modifications and refinements in the past (Telania, 2004). The four primary levels of evaluation are illustrated in the Figure 2 below.

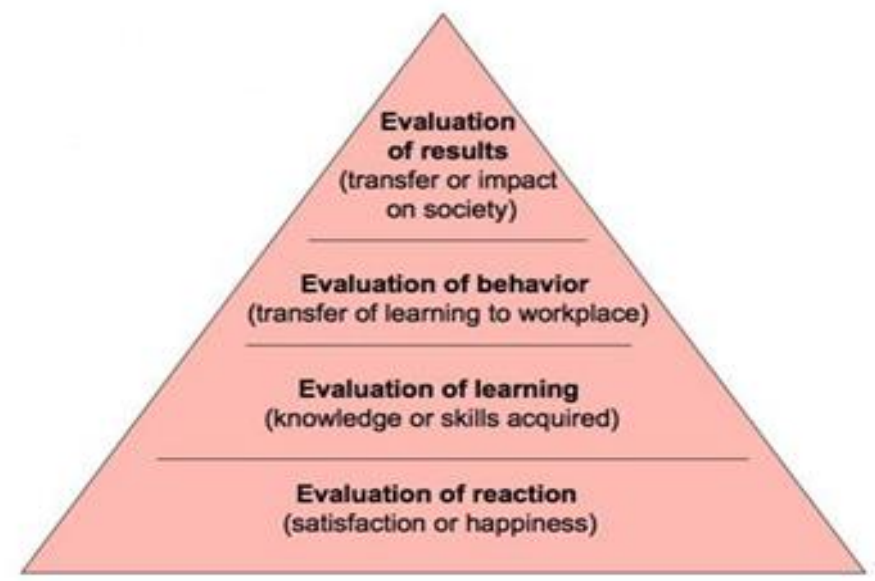

\section{Figure 2: Kirkpatrick's model of training evaluation}

Source: Kirkpatrick's Four-Level Training Evaluation Model (n.d.)

Kirkpatrick's model consists of 'Four Levels of Training Evaluation'. The four main levels are defined as follows (Kirkpatrick's Four-Level Training Evaluation Model, n.d.).

\section{Reaction}

This level measures how the people being trained and reacted to the training. It is considered that whether the people who were trained feel that the training was a valuable experience, feel good about the instructor, the topic, the material, its presentation, and the venue. Measuring the reaction helps understand how well the training was 
received by the audience. It also helps to improve the training for future trainees.

\section{Learning}

At level 2, it measures what the trainees have learned and how much has their knowledge increased as a result of the training.

\section{Behavior}

At this level, it evaluates how far trainees have changed their behavior, based on the training they received. Specifically, this looks at how trainees apply the information.

\section{Results}

At this level, the final results of the training are analyzed.

The level three of the Kirkpatrick Model does not totally depends on the first two levels of measurement. Employees may not apply what they learned however it does not mean that the training failed at levels 1 and 2 of the model. There are other affecting factors for employees to apply what they learned. They are, (a) the manager or supervisor can prevent employees from changing the way they do their jobs and (b) the employees might not have a desire to change their behavior. The level 3, which measures the behavior, can be the most difficult to measure effectively. The first two levels of the Kirkpatrick's Model provide immediate responses and results of the trainings. However, measuring the behavior requires more of a long-term approach. It can take weeks or even months to measure the behavior changes of employees that result from attending training sessions (How to assess the effectiveness of your training using the Kirkpatrick Model, n.d.). Kirkpatrick's model focuses mainly on immediate outcomes rather than the process leading to the results in the long run (Chong, 2006). 


\section{Conceptual framework of the study}

As informed by the literature review, the training cycle can be used as the model for evaluating the full process of the quality and food safety training at the ABC Company. Accordingly, the Kirkpatrick's training evaluation model is adopted as the conceptual framework of the present study. Thus, different evaluation levels explained in the Kirkpatrick's training evaluation model were incorporated in this conceptual framework in order to check the identified dimensions in the 360-degree analysis.

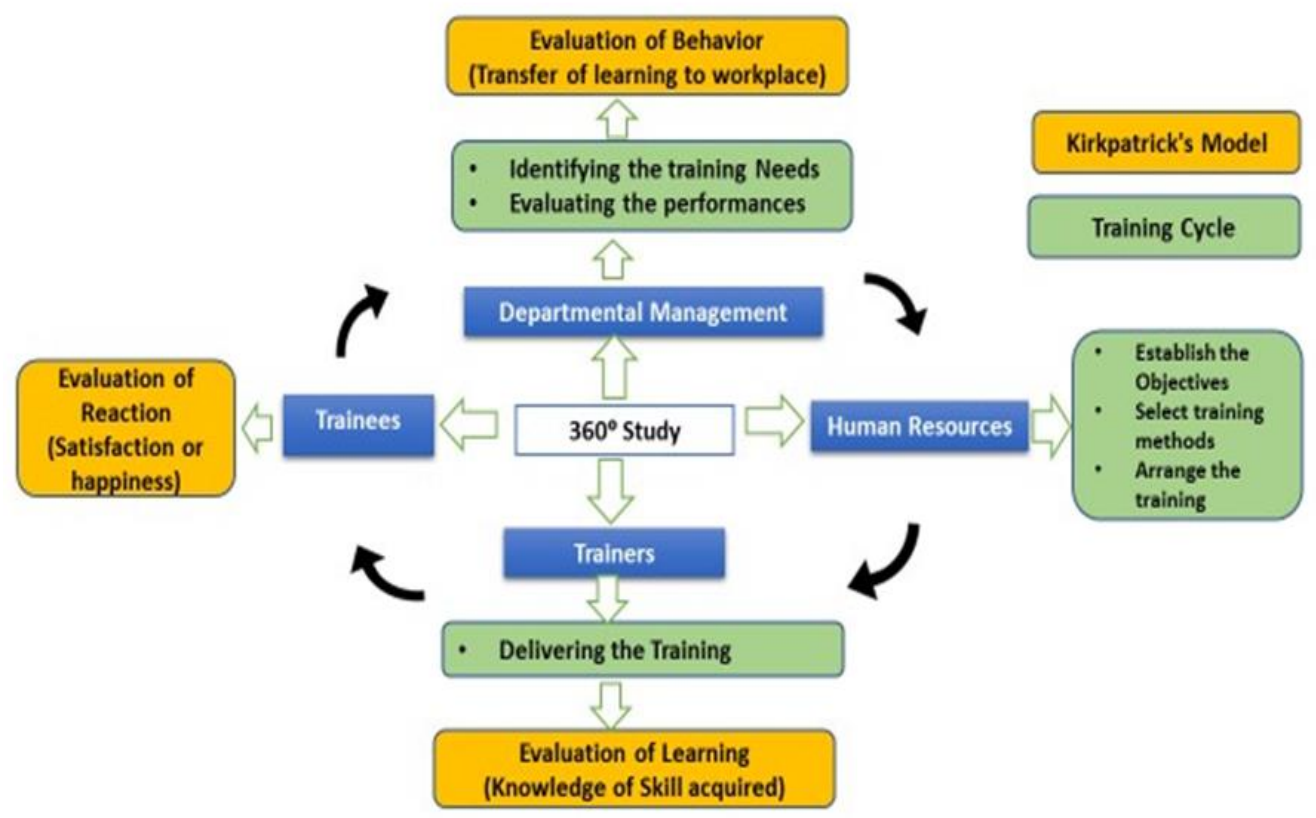

\section{Figure 3: Conceptual framework}

\section{Methods}

Adopting a deductive approach and following a predetermined framework based on the literature review, the study objectives were achieved in a qualitative inquiry. The exploration covered existing training process at the case organization in a 360 
degrees perspective which encapsulates the entire training process of the quality and food safety trainings, covering all major parties who involved in the process.

\section{Departmental Management}

\section{Human Resources}

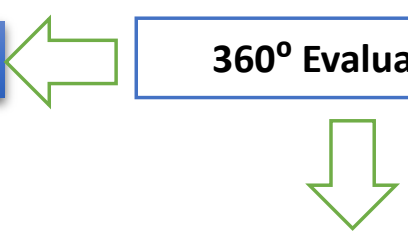

Trainers

Figure 4: Dimensions (parties) involved in the training process

The data were gathered through a questionnaire survey conducted for trainees and a structured interviews conducted with the trainers, human resource (HR) department and the heads of line departments (manufacturing and quality assurance). The questionnaire in English language was adopted for the trainee executives and the questionnaire in Sinhala language (translation) was used for the trainee non-executives.

The respondents of the study included the trainees, trainers, the HR executive, and the heads of line (manufacturing and quality assurance) departments. Accordingly, a sample of forty (40) out of the nighty (90) trainees in the non-executive grade were conveniently selected as the sample for collecting data through the questionnaire survey while all the nineteen (19) executives participated in the same trainings were considered to collect the data through the same questionnaire. (Twentytwo trainees who attended the same training were casual workers and they were not available in the carder currently). The external trainers 
those who conducted the external quality and food safety trainings and the internal trainer who conducted the internal trainings were interviewed. (The other internal trainer who conducted trainings in these training programs has left the company). The Human Resource (HR) executive who is responsible for the training process, and the heads of the two line departments (manufacturing and quality assurance) to which trainees belong were also interviewed.

The primary data were gathered through the questionnaire survey from the trainees and on one-on-one semi-structured interviews held with the rest of the respondents in the four dimensions of the training process. The semi-structured interviews were adopted in order to maintain the uniformity in the data gathering process. Interviews with the internal trainer, departmental management and HR executive were recorded and transcribed for in-depth reviewing and analyzing with the permission of the respondents. The names of the respondents being omitted to ensure the anonymity. Prior approvals were obtained from the interviewees and the appointments sought in order to carry out the interviews. The administration of the interviews was performed by the researchers with the support of an assistant. A summary of the data collection protocol is presented in the Table 2 below. 
Table 2: Method of data collection

\begin{tabular}{|c|c|c|}
\hline $\begin{array}{c}\text { Dimensions in the } \\
\text { Model }\end{array}$ & Respondents & Method \\
\hline \multirow[t]{2}{*}{ Trainees } & Executives & $\begin{array}{c}\text { Questionnaire } \\
\text { survey }\end{array}$ \\
\hline & Non-executives & $\begin{array}{c}\text { Questionnaire } \\
\text { survey }\end{array}$ \\
\hline \multirow[t]{2}{*}{ Trainers } & Internal & Interviews \\
\hline & External & Interviews \\
\hline \multirow[t]{2}{*}{ Department heads } & $\begin{array}{c}\text { Manufacturing } \\
\text { Department } 1 \text { and } 2\end{array}$ & Interviews \\
\hline & $\begin{array}{c}\text { Quality Assurance } \\
\text { Department }\end{array}$ & Interviews \\
\hline Human resources & HR Executive & Interviews \\
\hline
\end{tabular}

Data collected from the trainees through the survey questionnaire were obtained on a Likert scale and analyzed statistically. Thematic analysis was conducted for analyzing the qualitative data obtained through interviews.

\section{Findings and analysis}

Why quality and food safety training of the company has been ineffective

The analysis of data on the reasons why quality and food safety training of the company has been ineffective have been analyzed below in line with the four dimensions (trainee, trainer, heads of departments and HR executive).

\section{Dimension of trainees - Executives}

Two of the nineteen (19) executives participated in the quality and food safety trainings in year 2015/16 and 2016/17, have left the company and 
Thirteen (13) out of seventeen executives who are available in the executive carder $(76 \%)$ responded the survey questionnaire.

The responses are mostly positive for all the questions probed into the reaction of respondents on the training programs, except for the questions those focused on their prior knowledge about the purpose of the training course and about successful application of what they learned. In total, from $76.9 \%$ to $100 \%$ respondents were either 'agree' or 'strongly agree' with the positive aspects of the training, except for the above two matters. See the Figure 5 for summary for responses in this regard.

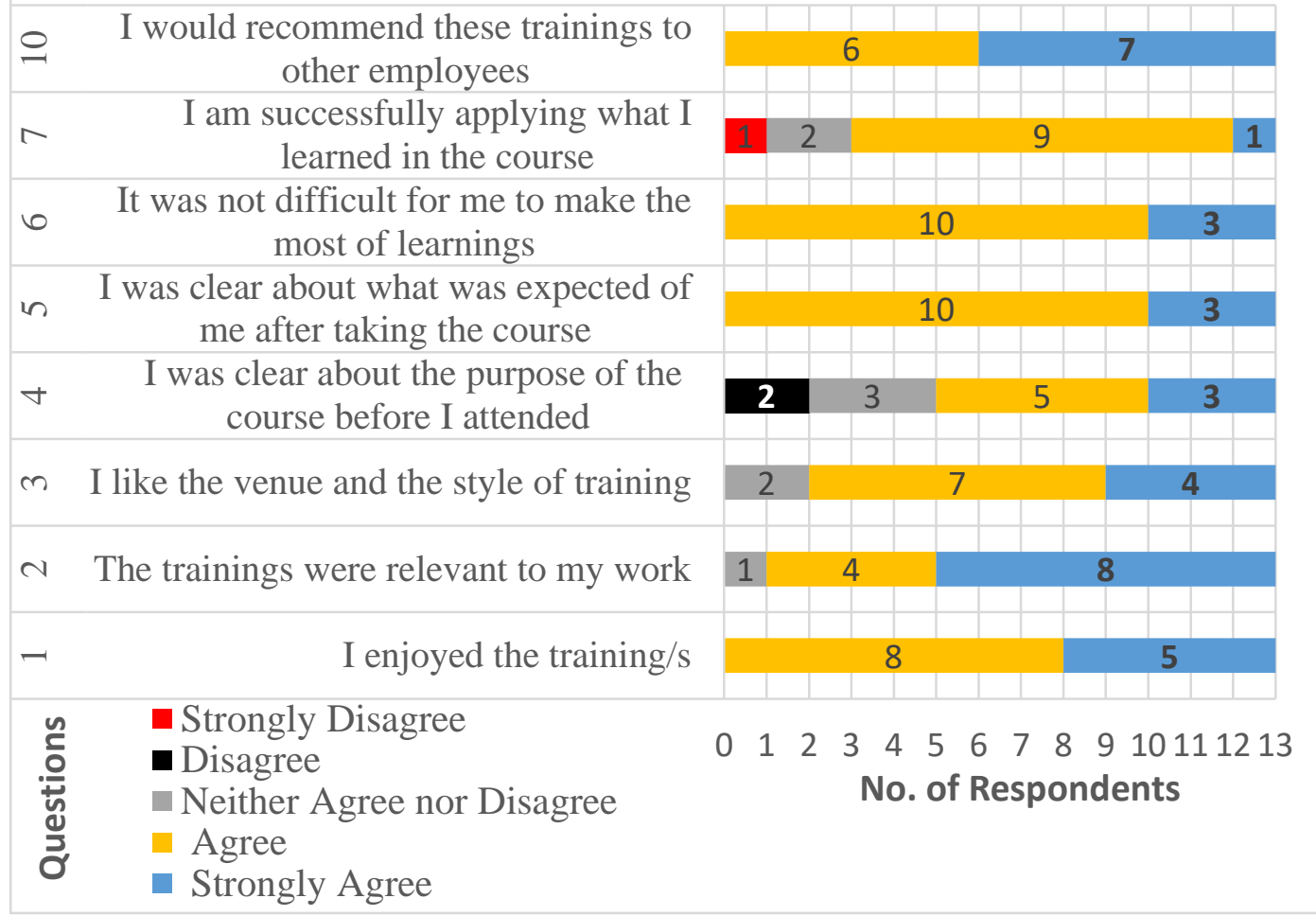

Figure 5: Summary of the responses of trainees- executives Source: Survey data (2018) 
However, while $23.1 \%$ trainee executives do not agree with the possibility of successful implementation of the learning achieved, the rest $(76.9 \%)$ of trainee executives have specified the reasons for successful implementation. The three major reasons identified in this regard were, (a) past experiences, (b) help from co-workers, and (c) help from the immediate supervisors. Hence, the responses are mostly positive on the matter whether the trainees knew about the purpose of the training course before they attended it. See the Figure 6 below for detailed data on this matter.

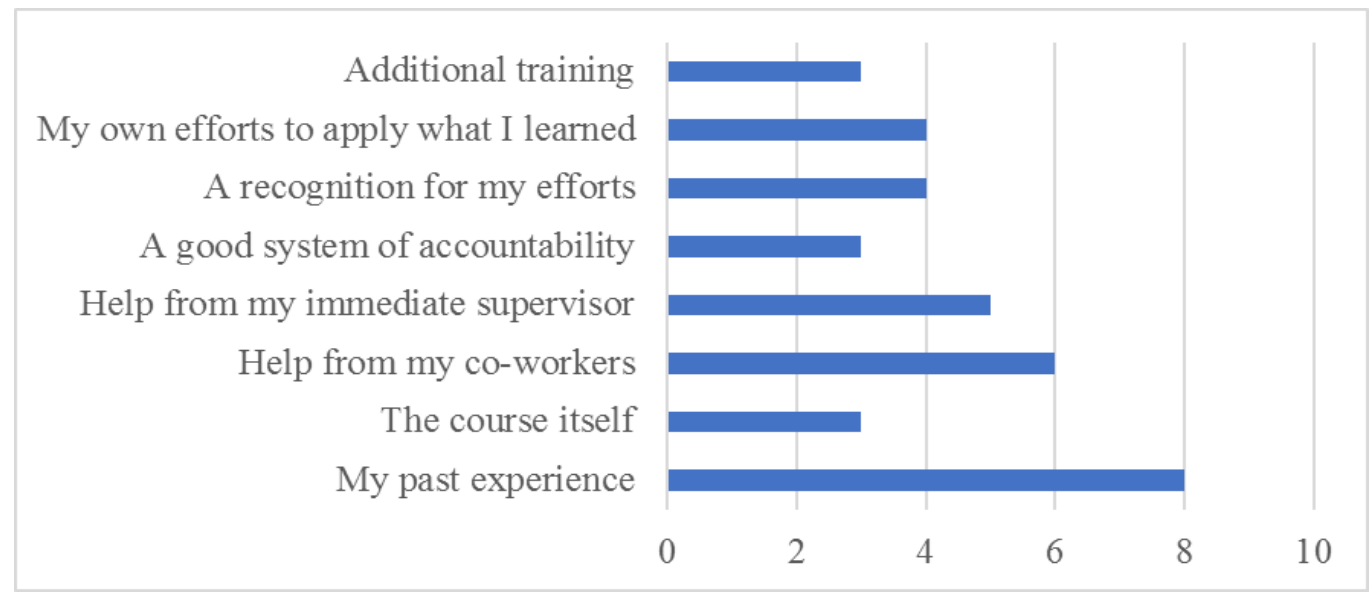

Figure 6: Reasons for successful implementation of what executives learned

Source: Survey data (2018)

Surprisingly, the executives have marked the lowest score for the reason of successful implementation which termed 'Training itself' which means, though they implemented what they have learned, that is mostly because of their experience and the assistance from the team and from 
the supervisor. Thus, the impact of the training itself in implementing what they have learned has been minimal. This confusing argument can be elaborated as, the executives trust their own experience than what they have learned at the implementation of something gained from the training.

\section{Dimension of trainees - Non-executives}

The category of non-executives comprised a mix of different levels of shop floor team members, ranging from the team leaders to the lowest level floor workers. Accordingly, some of the casual workers are also included in the sample. With regards to the sample of forty of the nonexecutives, only twenty-seven (67\%) have responded to the questionnaire. Ten out of those forty trained non-executives had left the company.

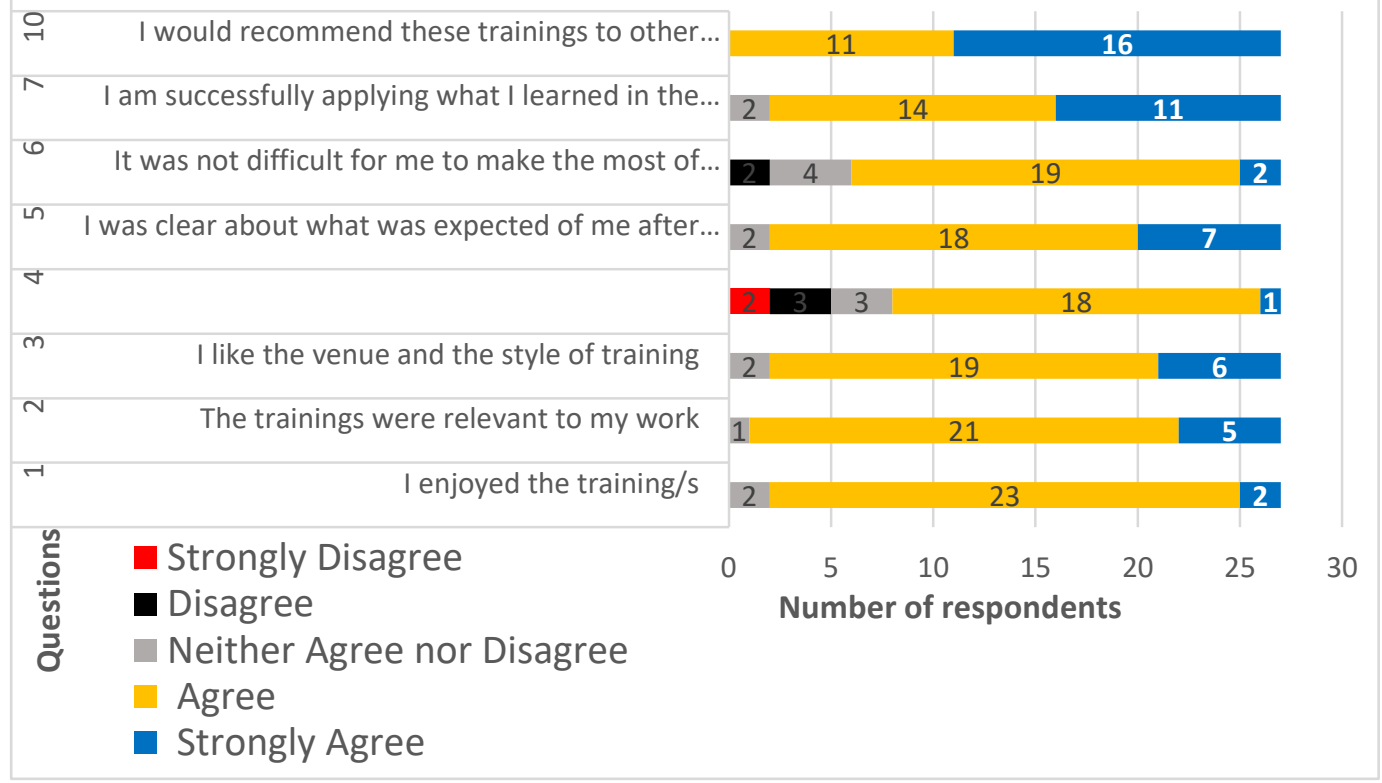

Figure 7: Summary of responses from non-executive trainees

Source: Survey data (2018) 
From among the non-executives, 92.6\% either agree or strongly agree that they are implementing what they have learned though the trainings. The other significant factor with this non-executive trainee group is, $22.2 \%$ of the participants were either disagree or neutral in accepting that the most of the trainings are not difficult for them to make a 6--learning. It means that $22.2 \%$ of non-executive participants had experienced difficulties in learning what was taught to them.

Comparatively, the non-executives have implemented what they have learned greater than that of executives did. However, it was found that the non-executives believe that the reason behind the implementation of what they learned is, the training itself together with the support of coworkers and their immediate supervisor.

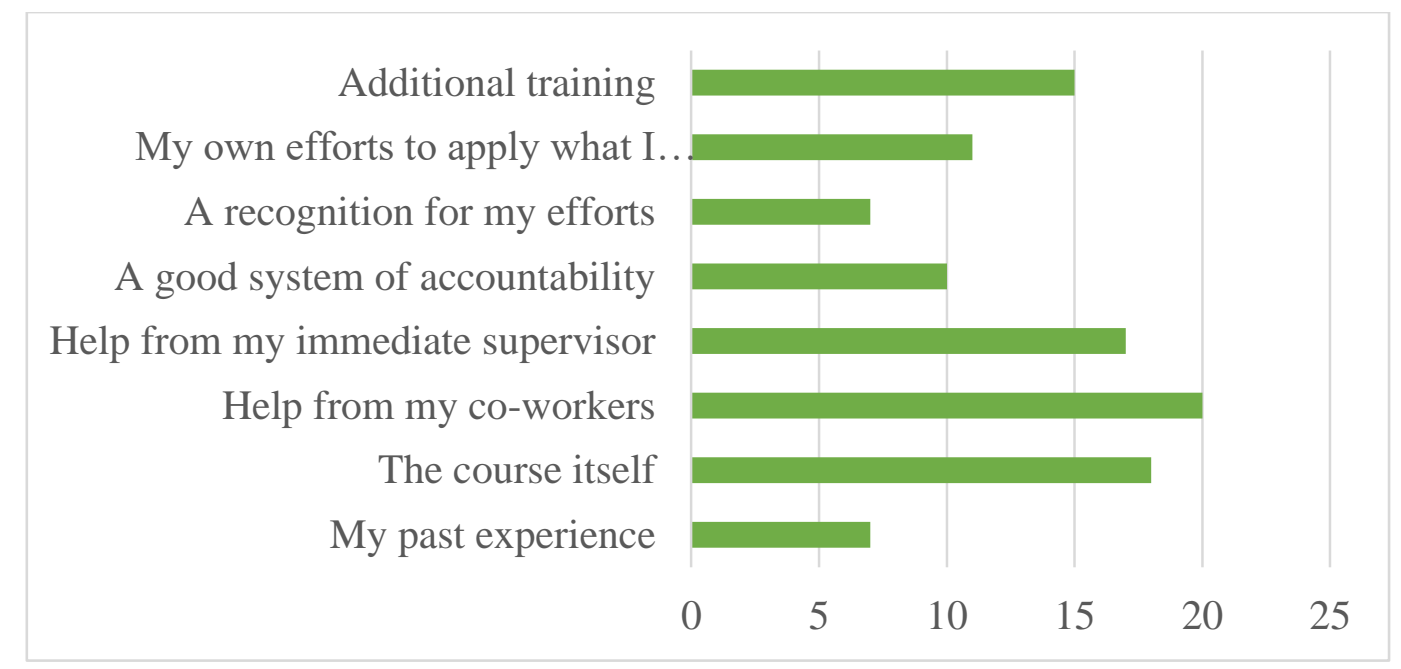

Figure 8: Reasons for successful implementation of what non-executives learned

Source: Survey data (2018) 


\section{Dimension of trainers}

An external Training Institute has facilitated external trainings and two trainers (A) and (B) have involved in conducting the three quality and food safety training sessions. During the interview of (A) and (B) separately, the both noted that the training programs for which the trainees participated from $\mathrm{ABC}$ company were common training programs. Those training programs were not customized (tailor-made) for the ABC Company. As per the list of trainees, $95 \%$ of participants are executives (Source: Training attendance master list of $\mathrm{ABC}$ Company - Year 2015/16, 2016/17 and 2017/18.)

The enrollment of the trainees from the $\mathrm{ABC}$ company was based on the request made by the $\mathrm{ABC}$ company. As further explained, $\mathrm{ABC}$ company has not discussed about the content of the trainings or about the competencies required for the participants in requesting the trainings for their employees. Both the trainers were in a common view about the participants' behavior during the sessions. Since these trainings had been conducted at least one year ago and the participants were from different organizations, the responders were not able to specify any specific remark about the active or interactive participation of the trainees of $\mathrm{ABC}$ Company. However, trainers assured that those trainings were interactive.

Further, both the trainers were not able to assure that the trainees of ABC Company learned what they intended to be taught. However, trainers have verified experiences to assure the effectiveness of their trainings delivered to the intended participants. The two trainers had a mixed view about the enhancement of the skills, knowledge and behavioral changes of the trainees occurred due to these particular trainings programs. Trainer (A) does not believe this occurrence while 
the trainer (B) does. The view of trainer (B) is that the skill, knowledge and behavioral enhancement of the trainees can be expected only through these trainings, however the refreshing trainings should be arranged to the same participants periodically.

As per the trainers, $\mathrm{ABC}$ company has not communicated about the competencies of the participants before the training and further had not requested any post training feedback as well. The Training Institute measures the post training feedback from each participant however, that information has not been shared with the ABC Company. The trainer (A) and (B) both agreed that the sustainability of the performance enhancement which is expected from the trainings may differ in the long run unless refreshment training is not arranged. However, the factors which can affect the job satisfaction can also impact on this long-term sustainability. Both the trainers were satisfied about the basic knowledge of the participants on the quality and the food safety.

The quality assurance manager (QAM) is the only internal trainer who is available with $\mathrm{ABC}$ Company currently. The QAM has conducted the trainings on the personal hygiene and the GMP (Good Manufacturing Practices) in the plant training room. These two trainings provide the required solid foundation in the food industry with respect to the improving of the basic quality and food safety awareness and practices. Hence, the outcome of these trainings initiates the basic attitude required for the quality and food safety assurances.

The trainer does not agree that the participants joined the training programs with an enthusiasm. Further, the actual number of the participants was lower than that of the expected/ planned numbers. Further, the trainer emphasized several times that the trainees participated in the programs since they were asked to do so and also to 
have a bit of relief from the work load. The trainer further mentioned that the low interest of trainees leads to disappointment of trainers in the program. The training programs are designed with many technical information and most of the participants were shop floor workers including the casual workers those who are not well knowledgeable on theoretical stuff. Adding further, the trainer commented that the worker grade carders do not know why the trainings are conducted and they are not in a position to gauge the value of the trainings. Hence, the course content itself might made the people low attentive for the trainings.

The content of the training is prepared by the trainer. The trainer always attempts to cover the basics of the quality and food safety practices and include the short videos mostly to grab the attention and interest of the trainees with a view to focusing the practical adaptation at the shop floor. Most of the time, those videos explain the improved quality and food safety practices which are not currently available in the plant. As an example, the trainer explained about the modern hand washing stations which are used in the food industry through the videos during the last GMP training session however, the hand washing stations which are available in the plant are very basic operations.

The human resources department does not provide the background of the trainees or any other guideline for the expected trainings and only the date and time of the training organized have been informed to the trainer. After the trainings, the human resources department has not obtained the trainer's feedback. However, a format for the post training evaluation has been filled by the participants at the end of each training session. 
The trainer does not agree to accept that, most of the participants learned what they intent to learn. As the reason, the trainer again emphasized the low interest of the participants. The trainer has experienced the sessions are more interactives with the executives however, the non-executives are just listeners. The trainer seemed disappointed through his bad experiences and is having an issue on how to capture the interest of the participants. Finally, the trainer concluded the interview mentioning as "It is waste of my time".

In summary, the comments from the external and internal trainers about their trainings and about the trainees are totally different and the response of the trainees are also completely different at the internal and external trainings.

\section{Dimension of HR department}

The HR executive who is responsible for the trainings of $A B C$ Company was interviewed. The comments from the HR executive cover the entire training process of $\mathrm{ABC}$ Company and however, common for the quality and food safety trainings as well.

The preparation of the annual training plan is performed during the annual budget preparation. The process initiated by circulating a standard format by the HR executive among the department heads to receive the training needs identifications from each department. The department heads request all the training programs required for their department as per the operation of the department. In this format, the target groups such as executives, team leader, operates etc., and the numbers of employees nominated for the training are communicated to HR executive. The quality assurance (QA) manager provides the quality and food safety trainings required for the entire plant team including the 
specific trainings which are focused for the QA team as well. In the QA trainings, the QA manager categories the training requirements as internal and external. Except the QA manager, other department heads also request the common quality and GMP trainings such as trainings on GMP, hygiene as well.

However, it is understood that during this process, the department heads decide the training requirement by naming the training programs and nominating the number of participants. The role of HR is to finalize the training institutes for the external trainings and the internal trainers, arranging and coordinating the trainings. The requirement of establishing the training objectives has not been identified. Upon the receipt of the training requirements, the $\mathrm{HR}$ executive prepares the annual training plan for internal and external trainings. The related external training institutes are contacted, and the training prospects issued by those organizations are used to select the required external trainings and to compare the cost factor. The annual external training plan prepared for the $\mathrm{ABC}$ Company is subjected to the approval during the budget approval process.

The process of identifying the training methods is not available as per the HR Executive. The HR executive's view about the training needs identification is, "It should be performed by the department management". However, she further confirmed that the structured and documented training needs identification process is not available with the ABC Company.

In selecting the external training institutes for quality and food safety trainings, the HR executive should have to manage with the available limited training institutes and the training programs as well as with the 
cost factor. If the cost of external training is higher than the cost allocated in the budget, the few executives from the selected team are sent for the training and those trainees are utilized as the resource persons to train the rest of the team members selected for the same training. The training skills of these 'trainers' are not a factor considered in this process. However, the turnover of these trained executives, has become a barrier in continuing the internal trainings. At the end of the internal trainings, the trainer assessment forms are filled by the participants. As per the HR executive, however, the use of these information to evaluate the trainers is not been performed. Further, arranging the tailor-made external training for the nominated group of peoples in accordance with the training needs has not been practiced.

Only a single private Training Institute, has been deployed for the quality and food safety trainings for the period covered in this research. Being the accreditation body of the quality and food safety system certification of the $\mathrm{ABC}$ company, this Training Institute has been selected for conducting external trainings. The training evaluation process is being managed and that has been done through the departmental managements and through a questionnaire organized by HRD. As per the HR executive, the casual workers have become a major concern in quality and food safety related trainings. The varying number of casual workers which is from 20 to 100 depending on the production seasons are employed through a manpower agency in the shop floor. Refreshing of these casual workers with new members very frequently is a serious issue which the plant management experiences. With this high turnover, covering all the new casual workers at least with the basic GMP and hygiene trainings is not possible, and the basic 
awareness is managed for the new comers by using a set of printed instructions.

The HR executive explained her view further on the effectiveness of the training highlighting the failure of achieving the intended outcomes of the trainings which is because of the higher turnover of the trained executives. Most of the trained executives do not show an interest to share the knowledge after the external trainings as a trainer and the HR executive brought some examples to justify her comment. Some executives attend only for the external trainings and that is to add qualifications for their carrier and then leave the company. Furthermore, in relation with the GMP and hygiene trainings, specifically the HR executive mentioned through her 11 years' experience that the expected attitude change has not been gained though the same trainings have been continued for years. Even though HR executive's comments are common for the entire trainings process, which is under her responsibility, there are commonalities which is applied for the quality and food safety.

In summary of the interview with the HR, the following causes were identified as the gaps of the current training management process which directly impact on the effectiveness of the trainings organized: (a) training process is not operated through the set objectives, (b) lack of a set protocol (including, training needs identification, selecting of the training methods according to the training needs and participants, a protocol to evaluate and use the data collected at the post training evaluation), (c) budget limitations, (d) lack of skilled internal trainers and turnover of trained executives, (e) ineffective criteria of selecting the external trainers, and (f) frequent turnover of casual workers. 


\section{Dimension of heads of departments (manufacturing and quality assurance)}

The heads of the two-manufacturing departments and the department of quality assurance were interviewed for obtaining relevant data.

\section{Manufacturing departments}

The production manager-01, head of one of the two manufacturing departments interviewed endorsed the process explained by the HR executive. The training needs of the existing employees of this department are identified during the performance evaluation. The department head discusses with the new comers to identify their training needs. A set documented procedure is not available to evaluate the training effectiveness. However, the head of the department discusses with the employees randomly after coming back from the trainings to gauge the employee's knowledge gain. Further, during the routing performance evaluations, they are monitored to observe the improvements or new initiatives.

The head of this department (production manager-01) is satisfied with the outcomes of the external trainings of his employees since there were instances that the employees initiated new events such as introducing new check lists for monitoring the processes. The production manager01 has noted that the reason for the lower effectiveness of the trainings is significantly due to the higher degree of turnover of the executives and team leaders who leave the company, putting new or low experienced executives to run the operation. While commenting on the possible reasons for the deterioration of the quality and food safety indicators, the production manager emphasized that the frequent 
refreshing of the casual workers and improper training and awareness of the quality and food safety norms as the major reasons.

The production manager-02 of the next manufacturing department also explained the same process of identification of the training needs which was explained by the HR executive and the production manager-01. The production manager-02 highlighted a major gap of the internal trainings. Though the employees are nominated for trainings as the individuals or groups, the trainings on GMP, hygiene and food safety are arranged during the day shift, and so the employees who are in the night shift may miss such trainings. The shift change happens once a week and the possibility of arranging the same training in the next week is minimal.

The comment of the production manager- 02 which is on the evaluation of the training effectiveness was different to that of others. The feedback forms are not used consistently and there are not fixed time frames to evaluate the trainings through those feedback forms. However, the department management monitor the effectiveness during the operations of the trained employees. A set documented protocol has not been implemented for this matter. As per the production manager-02, the effectiveness of the training is very low and two major reasons can be highlighted for this inadequate performance. First is that the internal trainings have not been designed on the practical aspects and those trainings are theory-driven. Since the level of education of the shop floor workers are low, the theory-bound trainings are not fruitful. Second, point is related to the attitudes of the employees on the shop floor level. Although they are trained, they do not follow or implement what they learned. The production manager-02 also highlighted the employee turnover (especially executives and the next level) of contract 
workers as a major cause for low effectiveness of trainings. Further, the production manager-02 added another two points, that are the low attention of the top management on the training and development of the human factor and the budget restrictions which limits the arrangement of the required trainings.

\section{Quality assurance department}

The quality assurance manager (QAM) who was the internal trainer for quality and food safety was interviewed as the department head of QA. QAM's comments as the department head could be cross verified since he was the internal trainer as well.

The process of identifying the training needs and nominating trainees explained by the head of QA was the same as mentioned by the other heads of departments. However, the QA manager uses a different process to evaluate the training effectiveness while monitoring the employees during the operations. The QA team participates mainly in quality and food safety related trainings and after the external trainings, the QAM checks the training materials, discussed with the participants and ask them to teach back what they learned.

Although, the QAM is the internal trainer, as well as a head of department, he accepts that the training effectiveness is low. As a department head, the QAM's comment was "How much we train the people on quality and food safety, many of them do not comply with basic hygiene norms".

QAM remarked that it is not about the knowledge or skills of them towards the job, but it is about their attitudes, poor awareness of the quality and food safety risks and negligence which causes for the low effectiveness of the quality and food safety trainings. QAM further 
emphasized, "Even so much they are trained, they don't want to change their behaviors, and they still continue the same behavior as usual". While proposing the repeat and refresh trainings, the QAM emphasized the requirement of a proper system for identifying the training needs and the training evaluation. Furthermore, the QAM indicated that the recording of the negligence and violation of quality norms done by employees as well as paying attention to such negative aspects at the annual employee evaluation are required.

In summary, although some comments revealed in the interviews are commonly related to the overall training programs, the major essence of that and some specific causes highlighted are directly related to the lower effectiveness of the quality and food safety training programs organized by the $\mathrm{ABC}$ company. The highlights are as follows: (a) set protocols for needs identification and training evaluation are not available, (b) failure of training the casual workers is a major reason of deterioration of quality indicators, (c) current training programs are not capable of changing the employee's attitudes towards the quality and food safety, (d) training outcomes are not sustainable, (e) turnover of the trained employees, and (f) budget restrictions and limited management commitment on the training and development.

\section{Possible actions to improve the training effectiveness}

The analysis of data on possible actions to improve the training effectiveness from the four dimensions (Trainee, trainer, heads of departments and HR executive) are presented in the section follows. 


\section{Dimension of trainees - Executives}

The trainee executives proposed a few suggestions towards the improvement of the existing quality and food safety training of the Company. The main points can be summarized to note that (a) make the content of the trainings more practical rather than based on theory, (b) GMP, hygiene, quality and food safety trainings should be continued, (c) GMP and hygiene training should be for all the employees, (d) provide room, support and leadership for implementing what have been learned, (e) arrange frequent refresh trainings, (f) conduct the trainings according to the training needs, (g) evaluate the training feedbacks, (h) implement some quality tools such as quality circles, and (i) arrange more trainings related to the quality and food safety systems such as ISO 9001, 22000, HACCP.

\section{Dimension of trainees - Non-executives}

The non-executives of the Company have brought to the notice some of the improvements needed in the existing training system as to (a) incorporate more quality and food safety trainings in the programs, (b) GMP and hygiene trainings should be continued, (c) GMP trainings should be accompanied with the job tasks (e.g. machine operations), and (d) provide training for new comers and provide opportunity for sharing knowledge among already trained employees,

In summary, both the executives and non-executives have proposed to continue the trainings while the top majority requests the key and basic trainings in quality and food safety which are GMP and Hygiene to be continued. These two training programs are the group trainings which cover most of the employees and the same trainings have been proposed to continue to improve the effectiveness of the trainings. In other words, 
the teams propose the refresh and follow up trainings to improve the effectiveness of the trainings. However, the proposals on changing the attitudes of the employees, encouraging the implementations of learnings, be more practical in trainings and the trainers with training quality are the most highly noted other proposals to improve the effectiveness of the quality and food safety trainings.

\section{Dimension of trainers}

The external trainers of the Company suggested some strategies from their viewpoints. Thus, their suggestions were, (a) getting the most suitable candidates for the training, (b) performing training need identification and analysis, (c) performing post training evaluation by the company, (d) organizing customized external training (if $\mathrm{ABC}$ Company plan more than 10 participants), (e) involving heads of department in training need identification and post training evaluation, (f) properly communicating with training institute on their training requirements, (g) linking training with organizational/ departmental/individual objectives and performances, and (h) relating training (other than awareness training) needs to the career progress of the employees. The internal trainer proposed to prepare a plan to change the attitudes of the non-executives toward to value the requirement of being complied with the quality and food safety requirements of the plant.

\section{Dimension of HR department}

As per the HR executives of the Company, there are several improvements to be made in the existing trainings system. As revealed by them, (a) the training effectiveness should be linked to the related KPIs, (b) grouping employees with similar training needs by the line 
departments so that tailor-made training programs can be arranged for them and (c) employee turnover intention to be identified in advance before recommending them for training programs.

\section{Dimension of heads of departments}

In proposing to improve the effectiveness of the quality and food safety trainings, the requirement of conducting the follow up and refresh trainings were proposed. The major deficiency which the manager highlighted was, though some effectiveness of the trainings were observed after coming back from the trainings, in the long runs those employees come back to normal and he believes, through encouragement, follow ups and refresh trainings, the effectiveness of the trainings can be improved. Minimizing the classroom training up to $40 \%$ and redesigning the internal quality and food safety trainings to include $60 \%$ of practical and relevant training aspects is the major requirement which was proposed by the production manager-02 to improve the effectiveness of the training.

\section{Discussion of findings}

As per the trainees' comments, their team members and the supervisors supported them to implement the learnings. As explained by Telania (2004), this situation describes that the manager or supervisor can prevent employees from changing their way of applying what they learnt. However, the executives trust their experience in implementing what they learned while non-executives reason the training itself assists them to implement the learnings. This may be a new insight which has not been highlighted in the Kirkpatrick's model or in the other models discussed. 
By measuring the reaction of trainees, it can be understood how well the training was received by the trainees and it also helps to improve the training for future trainees ("Kirkpatrick's Four Level Training Evaluation Model," n.d.). However, in this study, if only the trainees' comments were considered which is level one evaluation of Kirkpatrick's model, it could be misleading. The cause for trainees', positive comments may be because of two reasons. One is, as the internal trainer explained, they get a relief from the work when participating for a training and other is, the non-executives might have biased in responding to the questionnaire.

As per the findings of this study, theory-bound course contents, the trainers' incapability, enormous knowledge and competency gaps among the participants are the major reasons for the low attentive approach of the non-executives during the internal trainings. Arranging the mix of executives and non-executives for a same quality and food safety trainings may results in significant gaps in their learnings. Since the human resources department does not drive the trainings with set objectives and required guidance to the internal trainer, the internal trainings are incapable of achieving the intended results. In explaining the fourth step of the training cycle, Spilsbury (1995) gave a similar comment about the trainers and their preparation as to that trainings should be conducted with adequate preparation with professional trainers and with good presentation skills. Further, it was noted that the trainer should be more of a facilitator than an instructor.

The level one which measures the reaction of trainees in Kirkpatrick model again explains that the trainee should feel the training was a valuable experience and should feel good about the instructor, the topic, 
the material, its presentation, and the venue. It has been observed that the behavioral changes observed do not persist long term. It means if some improvements are gained through the quality and food safety trainings, those are not sustainable in the long run. The quality and food safety trainings were short trainings and not periodic. A clear majority of the trainees proposed to continue the GMP and hygiene trainings. This is in line with what explained by Kraiger, Ford, and Salas (n.d.) that most of the training performances will not be visible in the short run.

The non-existence of the refresh trainings which was highlighted by all four types of respondents may be another reason for the failure of the long-term sustainability of the behavioral changes which are expected through the trainings. The trainings programs of quality and food safety are not capable of changing the attitudes of employees to value the quality and food safety requirements and improve the quality and food safety indictors since the non-availability of the trainings designed on the behavioral base to change their attitudes. Rowell et al. (2013) suggested that "It is important that these training materials not only improve employee knowledge, but also provide employees the needed behavior-based learning to change their action" (p.385).

The gaps identified in the training process during the study are not limited to the quality and food safety trainings of $\mathrm{ABC}$ Company. It is about the entire training process of the organization. The nonavailability of the well-defined protocol for identifying the training needs, non-scheduling of trainings on the set objectives or KPIs (Key Performance Indicators), institutions and trainers are restricted by the budget limitations, unavailability of professional internal trainers, and 
non-availability of well-defined process of training performance evaluation are all violations of all five steps of the training cycle explained by Spilsbury (1995). In the Kirkpatrick's model, it further explains that when the training sessions are going to be planned, it should be stated with set of specific learning objectives ("Kirkpatrick's Four Level Training Evaluation Model," n.d.).

Since the inability of complying with a standard process like the training cycle, it has not allowed to identify the specific requirement of having a separate plan for training the casual workers to mitigate the risk of untrained casual worker in the shop floor which becomes of paramount importance because of the frequent refreshing of the casual carder.

Though it appears that the budget restrictions as a major bottle neck in arranging the external trainings, the setting of the comprehensive processes such as training needs identification, evaluation of training effectiveness and build the capable internal trainers will not be restricted by the budgets and it can contribute to implement and continue the basic and essential quality and food safety internal training such as GMP and Hygiene trainings. The high turnover of the trained employees may limit the training effectiveness of the quality and foods safety which should be addressed separately.

\section{Concluding remarks}

The study found that the effectiveness of quality and food safety trainings are not adequate, hence, the quality and food safety training process at $\mathrm{ABC}$ Company does not deliver the intended results. The driving force of the entire training cycle and process of any organization is the HR department. However, the responsibility of employee training at the HR department of this Company has been limited to the 
preparation of annual training calendar and its mere execution. In addition to this, the Company has not identified the training and development as a subsystem of the entire organizational system in order to achieve the improvement of the performances of individuals sharpening of skills, knowledge, changing the attitudes and gaining more knowledge to enhance the performance of the employees which eventually helps the company improvement.

The issues identified in the entire training process of ABC company, justifies the poor delivery of the intended results of the training programs of quality and food safety. The continuation of this same process may bring inappropriate results to the organization in the future. Unless the employees' attitudes and behaviors are developed towards the requirements of the quality and food safety of this highly perishable food manufacturer, there is a high probability that there may occur deviations from the prescribed quality and food safety standards and non-compliances in the food regulatory systems in the Company. This failure may cause a significant damage to the brand names of the $\mathrm{ABC}$ Company. At this backdrop, present study provides several practical implications to the case company towards improving the effectiveness of quality and food safety training. First, it implies that a review of the current training process of $\mathrm{ABC}$ Company and establishment of a comprehensive training and development protocol to cover the full training cycle would help setting grounds for effective training programs in the company. Second, it shows that the quality and food safety trainings should be managed on the prior-set objectives and an evaluation model (such as Kirkpatrick model) should be adopted to evaluate each step of the training process implemented. Finally, it implies that linking the individual performance evaluation with a set 
KPI (Key Performance indicators) would facilitate the monitoring of behavioral changes of the trainee employees of the company.

This study has a few limitations. The casual work force shares a major portion in the shop floor carder. Because of the very frequent refreshing of the casual workers, the trained causal workers were limited in the sample used for the data collection. The high turnover of the executives was a limitation which restricted the sample size of the trained executives. The data collected from the internal trainers could be more impartial, if it was possible to collect the data from the other internal trainer who had left the company.

\section{References}

Antic, Z., \& Bogetic, S. (2015). Food Industry Workers' Attitudes on The Importance of Factors Affecting Foodstuff Quality Management. Journal of Engineering Management and Competitiveness (JEMC), 5(1), 29-39.

Armstrong, M. (2009). Armstrong's Handbook of Humaan Resources Management Practice (11th ed.). London and Philadelphia: Kogan Page.

Biech, E. (n.d.). The training cycle: An overview. Retrieved on July 19, 2018, through www.dummmies.com:

https://www.dummies.com/business/human-resources/employeeengagement/the-training-cycle-an-overview/

Bramley, P. (1997). Evaluating Training Effectiveness, Translating

Theory Into Practic. Maidenhead, Hyderabad, India: Universities Press. 
Business Concepts- Human Resources. (n.d.). Retrieved on May 30, 2018, through mbaSKOOL.com: https://www.mbaskool.com/businessconcepts/human-resources-hr-terms/8685-training-anddevelopment.html

Chong, L. G. (2006, july 12). Evaluating Training Effectiveness: An Intergrated Perspective in Malaysia. Retrieved on May 20, 2018, through http://search.ror.unisa.edu.au/media/researcharchive/open/99159600172 $01831 / 53112394890001831$

Daud, Y., Jamaludin, K. R., \& Ramanr, J. V. (2012, October 30).

Human Factor Issue in Quality Management. Retrieved May 15, 2018, through file:///C:/

Users/HN30079/Downloads/JT_SE_59nov2012_KeluaranKhas2_07.pd)

De Feo, J. A., \& Juran, J. M. (2010). Juran's Quality HandBook (6th ed.). United States of America: McGraw-Hill.

Dewsnap, D. (n.d.). The Principals of Quality. Retrieved on May 12, 2018, through http://www.principles-of-

quality.com/qualityisanattitude.html

Donald Kirkpatrick's Learning Evaluation Model 1959; review and contextual material. (n.d.). Retrieved May 10, 2018, from www.businessball.com: https://www.businessballs.com/facilitationworkshops-and-training/kirkpatrick-evaluation-method-2049/\#toc-5

Gaungoo, Y., \& Jeewon, R. (2013, August 13). Effectiveness of Training Among Food Handlers:. Retrieved on May 10, 2018 through http://www.foodandnutrition 
journal.org/volume1 number1/effectiveness-of-training-among-foodhandlers-a-review-on-the-mauritian-framework/

Kirkpatrick's Four-Level Training Evaluation Model. (n.d.). Retrieved May 05, 2018, from Mind Tools:

https://www.mindtools.com/pages/article/kirkpatrick.htm

Kjeldsen, S. (2006, July). Training Impact Assessment of the United Nations Civil-Military Coordination. Retrieved May 15, 2018, from https://www.unocha.org/sites/dms/Documents/Training_Impact_Eval__CMCoord_-_FINAL_REPORT_(with_Annexes).pdf

Kraiger, K., Ford, K. J., \& Salas, E. (n.d.). Application of Cognitive, Skill-Based, and Affective Theories of Learning Outcomes to New Methods of Training Evaluation. Retrieved on June 10, 2018, through https://www.researchgate.Net/ publication/201381982_Application_of_Cognitive_SkillBased_and_Affective _Theories_of_Learning_Outcomes_to_New_Methods_of_Training_Eva luation

Manual on Training Evaluation. (n.d.). Retrieved June 10, 2018, from https://www.jica.go.jp/project/cambodia/0601331/pdf/english/5_Trainin gEvaluation.pdf

Morrison, J. E., \& Hammon, C. (2000, October). On Measuring the Effectiveness of Large-Scale Training Simulations. Retrieved on June 10, 2018 through Defence Technical Information Center: http://www.dtic.mil/dtic/tr/fulltext/u2/a394491.pdf

Rowell, A., Binkley, M., Thopson, L., Burris, S., \& Alvarado, C. (2013, November - December). The Impact of Food Safety Training on 
Employee Knowledge of Food Safety Practices for Hot/Cold Self-serve Bars. Retrieved May 10, 2018, from http://www.foodprotection.org/files/food-protection-trends/Nov-Dec13-Rowell.pdf

Santacruz, S. (2016, June 27). What is Food Safety? Retrieved on July 5, 2016, from Australian Institute of Food Safety: through https://www.foodsafety.com.au/resources/articles/what-is-food-safety

Spilsbury, M. (1995). Measuring the Effectiveness of Training. London: The Insitute for Employment studies.

Spindelndreier, D., \& Lesmeister, F. (2012, August). The Human Factor - What set Quality Leaders in Manufacturing Apart. Retrieved from The Boston Consulting Group (BCG): http://imagesrc.bcg.com/Images/what-sets-quality-leaders_tcm9-187857.pdf

Sprenger, R. (2009). Hygiene for Managers (15th ed.). South Yorkshire: Highfield.

Telania, E. (2004). How to Assess the Effectiveness of Your Training Using the Kirkpatrick Model. Retrieved on May 05, 2018, through https://www.eleapsoftware.com/files/wp/Kirkpatrick-eLeaP-AssessTraining.pdf 